griping pain and quite beyond his control, and with it the patient loses vast quantities of water and electrolytes. Vomiting is common at this stage, effortless and painless, and both vomit and stool are teeming with vibrios. Untreated, this loss of fluid and electrolytes leads within hours or at most a day to dehydration, with loss of skin elasticity, sunken eyes, dry mouth and tongue, and oliguria. It is not long before such dehydration leads to shock, with collapse of blood pressure, rapid shallow respiratory cyanosis, and clammy skin, muscle cramps, and anuria. Unrelieved, the patient rapidly dies, but with skilful treatment the dehydration can be overcome and recovery is speedy.

For a patient with the classical symptoms of cholera laboratory confirmation is scarcely necessary for clinical diagnosis. For the mild or symptomless case there is no other way to diagnose the infection, and as soon as cholera is suspected the co-operation of laboratory staff should be sought. A carefully taken rectal swab can be used for immediate dark-ground microscopy or for fluorescent antibody testing. The former may give a positive result in a few minutes, the latter in an hour or two. From the same swab $V$. cholerae may be grown in nutrient media overnight. The organisms are easy to identify in the laboratory. The difficulty is first to identify the infected patient; the only clue in the mild case lies in the patient's movements. Has he recently come from a cholera area? If he has, then a mild gastrointestinal upset may be due to infection with $V$. cholerae, and laboratory tests are essential to confirm the diagnosis.

\section{Traps in Tuberculosis}

The complications of syphilis and tuberculosis are no longer commonly seen in the neurological wards of our hospitals, their place being taken by the unusual cases of cryptogenic carcinoma, the collagenoses, or metabolic disease. They are thus in danger of being overlooked, for these diseases are still to be seen even if the ways in which they commonly present have altered. In the immigrant population the neurological lesions due to tuberculosis in particular are being seen more frequently again and should come high in the differential diagnosis of obscure meningitides and of spinal syndromes which may be accompanied by a suggestion of meningeal irritation. R. S. Kocen and $M$. Parsons ${ }^{1}$ have recently collected a number of such cases and point out that, whereas typical tuberculous meningitis is unlikely to be misdiagnosed, the same disease may present in a manner confusing to most clinicians yet equally responsive to treatment and equally tragic if unrecognized.

To understand the different types of syndrome one has only to go back to the well-known conception of the Rich focus $^{2}$ and realize that a tubercle, not necessarily part of miliary tuberculosis, if situated in the meninges can cause typical meningitis; if in the cerebral cortex, focal fits, and so resemble a cerebral tumour; or if in the cord itself a paraplegia. These early lesions may then be followed by meningitis if the focus later ruptures into the subarachnoid space. $\mathrm{K}$. B.
Taylor, H. V. Smith, and R. L. Vollum ${ }^{3}$ showed that the onset of tuberculous meningitis in some cases could be very abrupt, with an unexpectedly high polymorphonuclear count in the cerebrospinal fluid, and that this could settle spontaneously as what was essentially a tuberculin reaction subsided, giving the impression of cure, or perhaps of response to some antibiotic other than streptomycin. Such patients might be sent home only to be readmitted with the inevitable, and sometimes fatal, relapse. They stressed then the necessity for careful examination, repeatedly if necessary, of the cerebrospinal fluid for tubercle bacilli by all available means, and this needs to be re-emphasized now.

Paraplegia is a familiar complication of Pott's disease, but tuberculomata may be present in the spinal cord or the epidural space while routine $x$-rays appear normal, and sizeable epidural granulomata may be present despite apparently normal films if the lesion is confined to the laminae or pedicles. ${ }^{4}$ These are certainly rare. C. Arseni and D. C. Samitca ${ }^{5}$ found only nine intraspinal tuberculomata in a 20year experience of 38,500 neurosurgical patients drawn from a population at high risk, compared to 201 intracranial tuberculomata, while other spinal tumours were 43 times as common. Yet a progressive paraplegia accompanied by or followed by a meningitic reaction must arouse suspicion of this disease immediately, and it may be very responsive to antituberculous treatment. Other cases may simulate spinal metastases or, if in the lumbar region, even simple sciatica. A paraplegia may develop during the course of a recognized tuberculous meningitis. This may be due to an endarteritis and spinal infarction, or to organizing exudate strangulating the vascular supply to the cord, or again to an intramedullary tuberculoma.

R. A. Wiseman and A. Mahmood ${ }^{6}$ stress that these lesions are not so uncommon in countries where the disease is more prevalent than in Britain, and they may be seen more often here owing to the increase in movement of populations. One negative test of the cerebrospinal fluid does not exclude tuberculosis in obscure meningitis and paraparesis. A diagnosis of epidural secondaries cannot be accepted as certain without histological confirmation when there is no demonstrable primary malignancy. And it is worth remembering that the cerebrospinal fluid in the early stages of tuberculous infection may show little change in Mantoux-negative patients or in patients who are on steroids. Sir Hugh Cairns and Honor Smith ${ }^{7}$ drew attention to this 18 years ago, but the lesson deserves to be borne in mind.

Kocen, R. S., and Parsons, M., Quarterly fournal of Medicine, 1970, 39, 17 .

2 Rich, A. R., and McCordock, H. A., Bulletin of the fohns Hopkins Hospital, 1933, 52, 5 .

3 Taylor, K. B., Smith, H. V., and Vollum, R. L., fournal of Neurology, Neurosurgery, and Psychiatry, 1955, 18, 165.

4 Griffiths, D. L., Seddon, H. J., and Roaf, R., Pott's Paraplegia. London Oxford University Press, 1956.

Arseni, C., and Samitca, D. C., Brain, 1960, 83, 285.

6 Wiseman, R. A., and Mahmood, A., British fournal of Clinical Practice, 1967, 21, 13.

' Cairns, H., and Smith, H. V., in Modern Practice in Tuberculosis, ed. T. H. Sellors and J. L. Livingstone, vol. 2, p. 353. London, Butterworth, 1952. 